Urología N eurológica y Urodinámica

Arch. Esp. Urol., 58, 9 (915-924), 2005

\title{
TRATAMIENTO QUIRÚRGICO AMBULATORIO DE LA INCONTINENCIA URINARIA DE ESFUERZO FEMENINA.
}

\author{
Pedro $N$ a valón Verdejo, Cristóbal Zaragozá Fernández ${ }^{1}$, Felipe 0 rdoño Domínguez, Francisco \\ Sánchez Ballester, Joaquín Juan Escudero, M ilagros Fabuel Deltoro, Luis De la Torre Abril y \\ Emilio López Alcina.
}

Servicio de Urología. Unidad de CMA ${ }^{1}$

Consorcio Hospital G eneral Universitario. Valencia. España

\begin{abstract}
Resumen.- O BJETIVO: Evaluar los resultados obtenidos en el tratamiento quirúrgico ambulatorio de la incontinencia urinaria de esfuerzo genuina (IUE), durante los cinco años de integración del Servicio de Urología en la Unidad de Cirugía M ayor Ambulatoria (UCMA) de nuestro Consorcio Hospital General Universitario de Valencia (CHGUV).

MÉTODO: Entre enero de 2000 y diciembre de 2004 , hemos intervenido en la UCMA del CHG UV a 26 pacientes (49-78; media 69,8 años) afectas de IUE, mediante la colocación de cabestrillo suburetral sin tensión (TVT), realizado con anestesia local-sedación y en régimen estrictamente ambulatorio. Todas las pacientes fueron evaluadas mediante clínica y urodinamia, excluyéndose a las que presentaron algún tipo de prolapso concomitante genital, urinario o entérico y las que no cumplían requisitos sociales para CMA. No se excluyeron las que habían recibido con anterioridad
\end{abstract}

Pedro $N$ a valón Verdejo

Paseo Alameda 38 - 2

46023. Valencia. (España)

e-mail: pedronavalon@yahoo.com

Trabajo recibido: 19 de abril 2005 alguna otra técnica quirúrgica antincontinencia. Se evalúan los criterios de inclusión y alta, así como los resultados obtenidos y el grado de satisfacción mediante la elaboración de un cuestionario.

RESULTADO S: En 22 pacientes (85\%) se evidenció una IUE pura y en 4 (15\%) una mixta con predominio de esfuerzo. El 54\% (14 pacientes) fueron ASA I, 8 (31\%) ASA II y el $15 \%$ restante (4) ASA III bien compensada. La tolerancia a la intervención bajo a nestesia local (20$30 \mathrm{ml}$. lidocaína al 1\%) fue buena en todas las pacientes, habiéndose utilizado sedoanalgesia adicional (perfusión de propofol y remifentanilo) en 10 de ellas ( 38 $\%)$. El tiempo medio de intervención fue de 30 minutos (25-45) y el de permanencia en la UCM A hasta el alta de 100 minutos (80-140). Todas fueron dadas de alta tras la intervención. $N$ inguna precisó ingreso ni presentó retención urinaria tras la retirada de la sonda. En todas desapareció la IUE. Tres pacientes presentaron urgencia miccional postoperatoria que respondieron a los anticolinérgicos orales. Los resultados obtenidos son superponibles a los alcanzados con anestesia epidural e ingreso, siendo el grado de satisfacción con el tra tamiento recibido superior al $95 \%$.

CONCLUSIONES: El desarrollo de nuevos sistemas revolucionarios en el tratamiento de la IUE ha simplificado la cirugía de esta entidad de tal manera que podemos afirmar que un elevado porcentaje de pacientes afectas son candidatas a ser incluidas en un programa de CMA, mejorando ostensiblemente la relación costo-eficacia, no disminuyendo por ello la calidad asistencial ni el grado de satisfacción de las pacientes. 
Palabras clave: Cirugía Mayor Ambula toria. Incontinencia urinaria de esfuerzo. Anestesia local.

Summary.- O BJECTIVES: To evaluate the results of the outpatient surgical trea tment of genuine female stress urinary incontinence (SUI) over a five year period since the integration of the Department of Urology in the A mbulatory Surgery Unit at our hospital "Consorcio Hospital G eneral Universitario de Valencia" (CHG UV). METHODS: Between January 2000 and December 200426 patients (ages 49-78; mean age $69.8 \mathrm{yr}$ ) with the diagnosis of SUI underwent tension-free suburethral mesh sling (TVT) outpatient operations under local a nesthesia-sedation at the a mbula tory surgery unit of the CHGUV. All patients had clinical and urodynamic evaluation, excluding those presenting genital prolapse or non compliance with the social requirements for a mbulatory surgery. Previous anti-incontinence surgery was not an exclusion criterion. W e evaluate inclusion and discharge criteria, results and satisfaction degree measured by a questionnaire.

RESULTS: 22 patients (85\%) had genuine SUI and 4 (15\%) had mixed UI with predominance of the stress component. $54 \%$ (14) of the patients were ASA I, 31\% (8) ASA II, and $15 \%$ (4) well compensated ASA III. 0 peration tolerance under local anesthesia $(20-30 \mathrm{ml}$ $1 \%$ lidocaine) was good in all patients, having used additional sedation-analgesia (propofol-remifentanil IV perfusion) in 10 of them (38\%). Mean operative time was 30 minutes $(25-45)$ and stay at the unit discharge was 100 min. (80-140). All patients were discharged the same day. $N$ one of them required readmission or presented urinary retention after catheter removal. SUI disappeared in all of them. Three patients presented postoperative urge incontinence responsive to oral anticolinergic drugs. 0 ur results are similar to those obtained with epidural anesthesia and hospital admission, being the degree of satisfaction with treatment higher than $95 \%$.

CON CLUSIO N S: The development of new, revolutionary systems for the treatment of SUI has simplified the surgical trea tment of this entity, so that we can say a high percentage of patients may be included in an ambulatory surgery program, significantly improving costefficacy without diminishment of health-care quality or patient satisfaction.

\section{Keywords: A mbulatory surgery. Stress urinary}

incontinence. Local anesthesia.

\section{INTRODUCCIÓN}

Los profesionales de la sanidad hemos ido adquiriendo un interés creciente por conceptos como los de gasto sanitario y coste-efectividad de los procedimientos quirúrgicos. En este sentido, y en su implantación en la cultura asistencial quirúrgica de nuestro país, la Cirugía Mayor A mbulatoria (CMA) ha venido jugando un importante papel, sobre todo en los últimos años, cifrándose ya en 1998 la proporción de intervenciones quirúrgicas realizadas en régimen a mbulatorio, -según datos del IN SA LUD- en el $34 \%$ en Baleares, el $29 \%$ en Castilla-La Mancha, el $27 \%$ en Murcia y el $26 \%$ en Madrid (1).

La CMA se define como la atención a procesos susceptibles de cirugía realizada con anestesia general, local, regional o sedación que requieren cuidados postoperatorios poco intensivos y de corta duración, por lo que el paciente no necesita ingreso hospitalario y puede ser dado de alta pocas horas después del procedimiento (2). A unque ha sido denominada de diferentes formas: "cirugía sin ingreso", "cirugía de alta precoz", "cirugía de día", etc, el término más utilizado en España es el de Cirugía "Mayor" A mbulatoria, para diferenciarla de la cirugía "menor" que incluye procedimientos de muy baja complejidad y que, por lo general, nunca se han realizado con ingreso hospitalario, asociándose habitualmente a la anestesia local.

El incremento del nivel cultural, del denominado "estado de bienestar" y de la longevidad de la población, junto a una mayor "oferta" asistencial, están comportando un progresivo aumento de la demanda quirúrgica, poniendo en evidencia la limitación de los recursos sanitarios. La CMA surge con la finalidad de optimizar estos recursos, no solo agilizando los actos quirúrgicos y racionalizando los crecientes gastos sanitarios, sino también mejorando la calidad asistencial $(3,4)$.

La urología es una de las especialidades con mayor proyección en el campo de la CMA, pues su alto porcentaje de patología de mediana complejidad, susceptible de ser intervenida sin ingreso, y el espectacular avance de su arsenal tecnológico (endoscopia, ondas de choque, hipertermia y termoterapia, láser, etc.) han propiciado una evolución hacia procedimientos quirúrgicos cada vez menos invasivos, más 
simples y seguros, y, por lo tanto, con menos necesidad de hospitalización (5).

A unque los tratamientos urológicos incluidos "oficialmente" (2) en España, se limitan a la vasectomía, cistoscopia, dilataciones uretrales, litotricia extracorpórea, circuncisión, tratamiento de lesiones escrotales, orquidectomía, cirugía del hidrocele, orquidopexia y meatoplastia, sus posibilidades, como demuestran algunas experiencias $(6,7)$, son mucho mayores. La Conselleria de Sanitat Valenciana (8) publicó una relación que incluye mas de 30 tipos distintos de intervenciones, tanto de patología inguinoescrotal, peneana y uroginecológica, como de procedimientos endoscópicos y percutáneos.

La incontinencia urinaria de esfuerzo (IUE) se define como la pérdida involuntaria de orina por la uretra provocada por aumentos en la presión intraabdominal, que normalmente se neutraliza por el control neurógeno de la resistencia uretral y la disipación de las fuerzas intraabdominales, a través del armazón fisiológico de la uretra en el interior de la pelvis (9).

A unque existen más de 150 técnicas quirúrgicas para tratarla, los cabestrillos pubovaginales son considerados en la actualidad como el procedimiento de elección para la mayoría de pacientes, pues otros como las suspensiones con aguja no han superado la prueba del tiempo y las retropúbicas conllevan una mayor morbilidad (10).

La corrección de la IUE es un procedimiento quirúrgico frecuente y que ha simplificado su realización de tal manera, que un alto porcentaje de pacientes son candidatas a ser incluidas en un programa de CMA ya que la técnica quirúrgica cumple todos los criterios de inclusión recomendados en la Guía de O rganización y Funcionamiento para la CMA (1) que se recogen en la Tabla I. A simismo, el hecho de que pueda ser realizada con anestesia local o con localsedación, implica que tampoco sea necesario ser muy selectivos en lo que al riesgo quirúrgico y anestésico se refiere, lo que además la convierte en un procedimiento idóneo en pacientes añosas donde el riesgo a nestésico suele ser elevado.

Su carácter "benigno" es el responsable del trato "poco preferente" de su tratamiento, lo que acumula pacientes en lista de espera, determinando una morbilidad y repercusiones psicosociales, económicas y laborales que pueden y deben ser evitadas.

\section{PACIENTES Y MÉTODO}

Desde nuestra incorporación a la Unidad de CMA (UCMA) del Consorcio Hospital General Universitario de Valencia (Enero de 2000 - Diciembre de 2004), hemos intervenido de IUE mediante la colocación de cabestrillo suburetral sin tensión (TVT) con carácter ambulatorio a 26 pacientes con edades comprendidas entre 49 y 78 años (media 69,8).

Todas las pacientes fueron evaluadas clínica y urodinámicamente, presentando IUE pura 22 (85\%) y mixta con predominio de esfuerzo las 4 (15\%) restantes. Fueron excluidas las que presentaron un prolapso concomitante genital, urinario o entérico que obligase a su corrección en el mismo acto. No fueron excluidas las que habían sufrido previamente otra técnica quirúrgica antiincontinencia, circunstancia que se dio en 9 (35\%), realizándose en 6 una suspensión de cuello vesical por vía suprapúbica y en 3 una técnica de agujas.

El 54\% fueron A SA I (14 pacientes), el 31\% (8 pacientes) ASA II y el $15 \%$ (4) ASA III bien compensadas.

En todas se realizó el procedimiento mediante la siguiente secuencia operatoria:

1) Instilación de 5 cc de gel de xilocaína al $2 \%$ en la uretra y vagina unos 30-40 minutos previos a la intervención.

2) Posición de litotomía no superando los 60 de flexión. Colocación de sonda de Foley no $18 \mathrm{Ch}$.

3) Infiltración suprapúbica con anestésico local (20 y $30 \mathrm{ml}$ de lidocaína al 1\%) a ambos lados de la línea media, con una separación entre ambos puntos de unos tres traveses de dedo, sobre cada tubérculo púbico, utilizando una aguja larga (habitualmente $22 \mathrm{G}$ ) que nos permita la infiltración profunda por detrás de las ramas del pubis en dirección hacia la uretra, así como la infiltración en sentido contrario, es decir, desde la cara vaginal anterior a ambos lados de la uretra por detrás del pubis en dirección hacia el hipogastrio. Para esto último recomendamos curvar la 
aguja (unos 40-60 grados dependiendo de la anatomía de la paciente) con el fiador de la aguja insertado para evitar la obturación de su luz por la angulación de la misma (Figura 1).

4) Incisión de unos 0 ' $5 \mathrm{~cm}$ por encima de la sínfisis del pubis a ambos lados de la línea media, sobre los puntos infiltrados con anestésico.

5) Incisión sagital vaginal de unos 1'5 cm en uretra media a $1 \mathrm{~cm}$ aproximadamente del meato uretral externo y disección roma parauretral bilateral hasta la fascia endopélvica.

6) A través de la sonda vesical introducimos una guía tutor rígida, con lo que podemos desplazar la uretra y el cuello vesical durante la inserción de la aguja del TVT.

7) Desplazando la uretra al lado contrario al que vamos a pasar la aguja (aguja y sonda se cruzan) pasamos ésta desde la apertura vaginal hasta hipogastrio, repitiendo la maniobra en el lado contralateral. Ultimamente preferimos colocar el cistoscopio desde el principio, siendo éste el que desplaza la uretra y el cuello vesical cuando introducimos las agujas, evitando de esa forma los pasos de colocación y retirada de sonda así como de la guía del tutor.

8) Con las agujas colocadas, realizamos cistoscopia para descartar la perforación vesical o uretral.

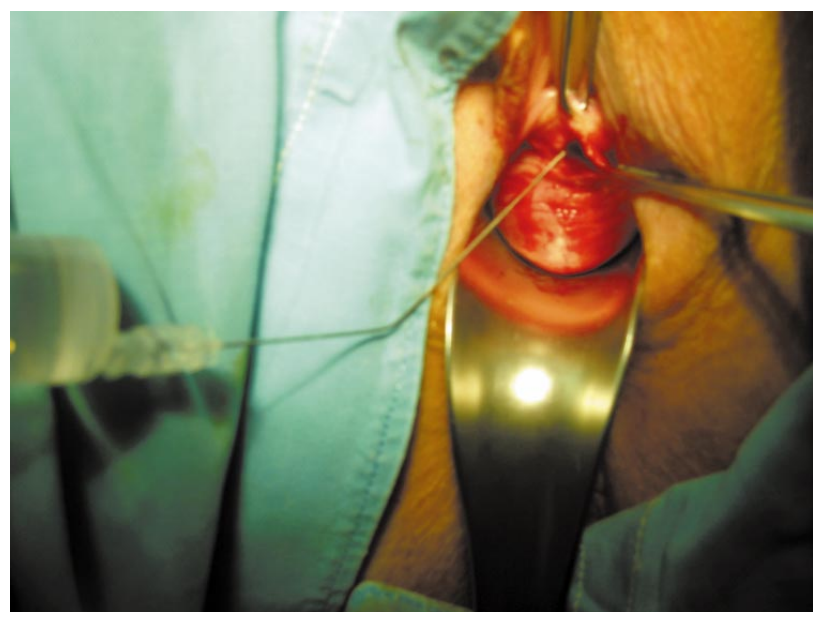

FIG URA 1. Infiltración anestésica del espacio retropúbico.
9) Tras la extracción de ambas agujas por hipogastrio, la cinta de polipropileno del TVT queda alojada a nivel suburetral (en tercio medio). Se retira la envoltura que recubre la malla, siendo recomendable interponer una tijera de Mayo entre la banda y la uretra mientras se tira de dicha envoltura con la finalidad que la cinta quede libre de tensión y no comprima la uretra (Figura 2).

10) Corte de la malla a ras de la piel abdominal y sutura de las incisiones hipogástricas y vaginal con uno 0 dos puntos sueltos de poligalactina $3 / 0$ (Vicryl $\left.\operatorname{rapid} \AA^{\circledR}\right)$.

11) Colocación de sonda de Foley 18 Ch y taponamiento vaginal que se retira a las 24 horas.

En todos los casos se realizó profilaxis antibiótica, utilizando una pauta monodosis (cefuroxima $750 \mathrm{mg}$ por vía intravenosa) previa al procedimiento quirúrgico.

El tratamiento postoperatorio recomendado consistió en la administración de analgésicos menores (metamizol magnésico por vía oral, hasta $2 \mathrm{~g}$ cada 6 horas).

Tras la intervención, todas los pacientes permanecieron en la Unidad de Recuperación Postanestésica (URPA) de la UCMA durante aproximadamente dos horas; y los controles posteriores se realizaron a las 24 horas, al mes y a los 3 meses. En las

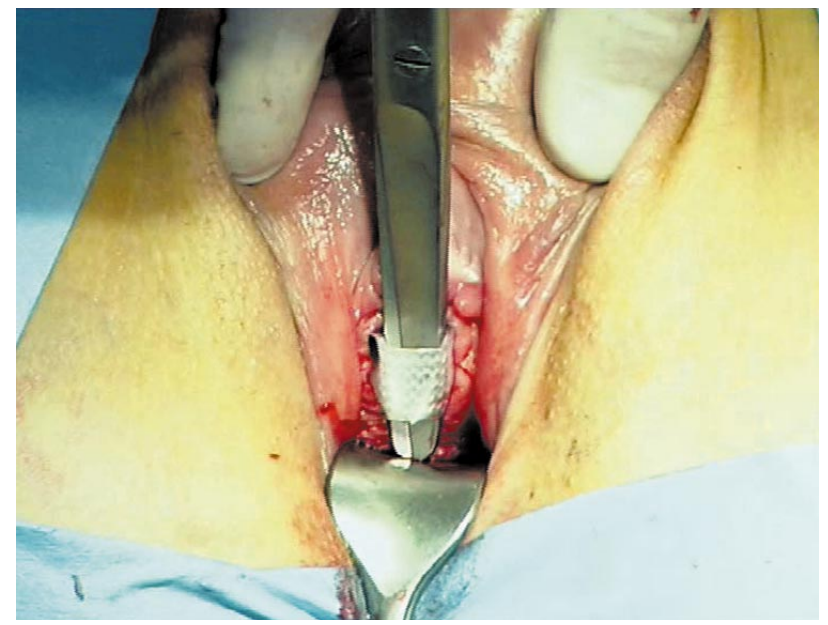

FIG URA 2. Interposición de tijera entre uretra y malla. 
TABLA I. CRITERIO S DE IN CLUSIÓN DE PRO C EDIM IEN TO S Q UIRÚRG IC OS

- No precisar preparación compleja

- Escaso riesgo hemorrágico

- Tiempo total de intervención inferior a 90 minutos

- Dolor postoperatorio controlable con analgesia oral

- No precisar drenajes con débito elevado

- Tolerancia oral precoz

- Que permita deambulación precoz

revisiones hemos realizado una breve encuesta anónima sobre el grado de satisfacción con el tratamiento recibido.

\section{RESULTADOS}

Todas las pacientes fueron intervenidas con anestesia local, precisándose entre 20 y $30 \mathrm{ml}$ de solución anestésica de lidocaína al 1\%. En 10 casos (38\%) fue necesario añadir sedoanalgesia mediante una perfusión de propofol y remifentanilo, motivado más por el estado de ansiedad de la paciente que por dolor 0 intolerancia al procedimiento.

El tiempo medio utilizado en la intervención quirúrgica fue de unos 30 minutos (rango 25-45).

Tras la intervención, las pacientes fueron trasladadas a la URPA donde permanecieron una media de 100 minutos (rango 80 - 140) a compañadas de un familiar y controladas por personal de enfermería hasta que la paciente cumplió los criterios de alta que apuntamos en la Tabla II. Todas fueron dadas de alta tras la intervención, no precisando ninguna de ellas ingreso hospitalario por complicaciones inmediatas intra ni postoperatorias.
N inguna presentó retención urinaria tras la retirada de la sonda uretral, manifestando todas la desaparición de la IUE. Tres pacientes (11\%) con urgencia miccional postoperatoria respondieron satisfactoriamente a los anticolinérgicos orales.

En la revisión a los tres meses, todas presentaron una evolución satisfactoria, contestando la globalidad una encuesta sobre el grado de satisfacción del tratamiento recibido, cuyos resultados se detallan en la Tabla III.

El seguimiento medio fue de $30^{\prime} 3 \pm 14^{\prime} 0$ meses (rango 3-58), habiéndose constatado en su totalidad la desaparición del componente de esfuerzo de su incontinencia. En 7 pacientes (27\%) que presentaron urgencia miccional (4 diagnosticadas preoperatoriamente y 3 con hiperactividad de novo) se controlaron los síntomas con la administración de anticolinérgicos orales.

\section{DISCUSIÓN}

En la última década los urólogos estamos asistiendo a una "revolución" en lo que se refiere a determinados aspectos relacionados con la IUE, tanto desde el punto de vista de su fisiopatología como de su terapia y de su enfoque médico o quirúrgico. Estos cambios han generado nuevas expectativas en los pacientes que sufren esta patología y en los médicos que la tratamos (11).

Han surgido nuevas teorías que cuestionan la clásica concepción de la IUE como mero producto de una alteración en la transmisión de presiones. Así, Petros y UImsten (12) proponen en su "teoría integral" que la pared vaginal anterior juega un papel fundamental en el balance del soporte del suelo pélvico. Según esta teoría, la incontinencia de esfuerzo y por

TABLA II. CRITERIO S DE ALTA

- Consciente y orientado
- Constantes vitales estables durante la última hora
- Tolerancia de líquidos
- Diuresis espontánea
- Control del dolor

- Deambulación autónoma sin sensación de inestabilidad

- Ausencia de sangrado

- Ausencia de nauseas y vómitos 
urgencia femenina tienen una etiología común, pues la laxitud de la pared vaginal anterior permite la activación de ciertos receptores de estiramiento en el cuello vesical y uretra proximal desencadenando un reflejo de micción inadecuado, lo que puede provocar inestabilidad del detrusor y micción imperiosa, polaquiuria y nicturia. La uretra se cierra en su porción media y no en el cuello de la vejiga, por lo que la falta de soporte de la uretra media y pared vaginal anterior por los ligamentos pubouretrales, junto con el deterioro de la función y la inserción de los músculos pubococcígeos, predisponen a que las pacientes padezcan incontinencia de esfuerzo. Posteriormente, De Lancey (9) publicó la "teoría de la hamaca" que de alguna forma complementa a la anterior y reafirma el valor de la uretra media en los mecanismos de continencia, pues afirma que la presión intraabdominal es transmitida a la uretra, cerrando su salida al ser comprimida contra los soportes de la aponeurosis pubocervical y la pared vaginal anterior.

A la luz de estas nuevas teorías sobre la fisiopatología de la IUE se han diseñado diversos sistemas de corrección quirúrgica basados en el empleo de cabestrillos sin tensión, tales como TVT (Gynecare), IVS (Tyco), Sparc / Monarc (AMS), Urotape / O ptape (Porges), Uretex (Bard), etc. que emplean mallas de implantación suburetral. Las diferencias entre estos sistemas radican básicamente en el tipo de malla empleado y el acceso para su inserción, pero todos ellos pretenden la misma finalidad: proporcionar un soporte adecuado a la uretra media. No obstante, los resultados no parecen depender exclusivamente de la técnica quirúrgica, ya que las características de la malla empleada posiblemente sean tan importantes o más que la propia técnica, no debiéndose extrapolar los datos relativos al TVT para otros dispositivos (13).

TABLA III. SATISFAC CIÓN CON EL TRATAM IEN TO RECIBIDO.

\begin{tabular}{|l|c|c|}
\hline CA LIFICA CIÓ N & No Casos & $\%$ \\
\hline Excelente & 21 & $81 \%$ \\
\hline Buena & 4 & $15 \%$ \\
\hline Regular & 1 & $4 \%$ \\
\hline Deficiente & 0 & $0 \%$ \\
\hline
\end{tabular}

El mecanismo de acción del TVT reside en la corrección del soporte inadecuado de la uretra debido a la alteración de los ligamentos pubouretrales y pared vaginal suburetral, creando una resistencia uretral dinámica durante el esfuerzo sin interferir la función uretral de reposo, no elevando el cuello vesical ni reducir su movilidad $(14,15)$, apareciendo un incremento en el índice de transmisión en la zona media de la uretra sin cambios en la presión uretral de cierre $(14,16)$; en consecuencia, es una técnica idónea para cualquier tipo de IUE, tanto en caso de hipermotilidad uretral como en la incompetencia uretral primaria, por lo que muchos autores consideran actualmente improcedente la determinación de la presión uretral de cierre o de la presión abdominal de fuga, cuestionando el valor del estudio urodinámico preoperatorio en la era del TVT, ya que los datos que aporta no suelen interferir en la decisión terapéutica, pudiendo únicamente informar acerca del pronóstico, pues una baja presión uretral de cierre 0 una presión abdominal de fuga inferior a $60 \mathrm{~mm} \mathrm{H} 20$ parecen asociarse a una menor probabilidad de buenos resultados $(17,18)$.

El hecho de que el procedimiento quirúrgico con TVT sea de fácil aprendizaje, mínimamente invasivo y con resultados satisfactorios a largo plazo $(15,19,20)$ ha hecho que su uso se haya generalizado de tal manera que se estiman en unas 200.000 las unidades implantadas desde que Ulmsten (21) describiera la técnica en el año1996, habiendo desplazado a la colposuspensión de Burch como el "patrón oro" con el que deben ser comparadas todas las demás técnicas quirúrgicas $(22,23)$. Los estudios comparativos con la técnica de Burch muestran unos resultados similares pero con un tiempo sensiblemente menor del acto quirúrgico, de la recuperación y del retorno a la actividad normal con TVT así como un coste económico mucho más bajo $(24,25)$.

A unque en este estudio hayamos establecido la presencia de prolapsos pelvianos como criterio de exclusión de las pacientes, pues creemos que su tratamiento se sale de la competencia de la CMA, la TVT puede emplearse junto a otras técnicas de reparación del suelo pélvico no aumentando por ello el número de complicaciones; tan solo produce un aumento del tiempo quirúrgico y de la estancia hospitalaria, pudiendo asociarse de forma segura y efectiva (26, 27). Tampoco parece haber un mayor riesgo de fracaso en las pacientes por razones de edad, menopau- 
sia, obesidad o cirugías asociadas (28, 29). Igualmente, los antecedentes de realización previa de otras cirugías antiincontinencia no parecen influir en los resultados ni en las complicaciones $(30,31)$ y en nuestra experiencia, tampoco encontramos mayor dificultad en la técnica quirúrgica.

En cuanto a la discusión referente a la utilización o no de sonda uretrovesical postoperatoria tras la colocación del TVT, consideramos innecesario su empleo sistemático, pero en CMA deben extremarse todas las medidas para minimizar riesgos de complicaciones y, por ello, creemos que su colocación y un taponamiento vaginal de 24 horas disminuye el riesgo de hemorragia y de hematomas, con una mínima morbilidad.

Por otro lado, y en los últimos años, la CMA está avanzando de forma imparable; suponiendo en Estados Unidos cerca del $60 \%$ de todas las intervenciones realizadas $(5,32)$. A simismo, son cada vez mas frecuentes los trabajos divulgados en las revistas de urología de nuestro país acerca de este sistema organizativo de la asistencia quirúrgica $(6,33)$, lo que pone de manifiesto que la urología es una especialidad con gran proyección en el campo de la CMA.

Coincidimos con Llopis y cols (7) en que la CMA constituye un sistema asistencial en el que todos los participantes se benefician. Para el hospital representa una reducción del coste sanitario que varía entre el 25 y $75 \%$ según diversos autores (34-36), y un mayor número de camas disponibles al no quedar los paciente ingresados, ya que según nuestra experiencia y la de otros autores, la estancia hospitalaria media de las pacientes intervenidas de IUE mediante la colocación de TVT con ingreso y anestesia raquídea o general varía entre 1 y 3 días $(37,38)$ ya que la estancia hospitalaria se prolonga frecuentemente por complicaciones derivadas de la técnica anestésica (nauseas, vómitos, cefalea, etc.) más que de la propia intervención. En lo que concierne al paciente, su modo de vida se altera mínimamente ya que vuelve a su domicilio a las pocas horas de ser intervenido, reduciéndose ostensiblemente el grado de ansiedad así como la probabilidad de complicaciones propias de la hospitalización, como la infección nosocomial y las secundarias al encamamiento (39). A demás, el grado de satisfacción suele ser elevado, tal y como demuestra nuestra encuesta, y que se sitúa en torno al $95 \%$. Para el cirujano, aunque al principio la CMA pueda ser considerada como una cirugía de segunda línea, pronto se ve implicado en un sistema de trabajo donde "todo vale" para minimizar riesgos, desde la instilación de gel de xilocaína en la uretra y vagina unos 30-40 minutos previos a la intervención, la metódica infiltración con anestésico local, la utilización de incisiones justas, hemostasia rigurosa, así como obviar gestos quirúrgicos innecesarios para abreviar el tiempo operatorio, etc., suponen un reto profesional, que al menos para nosotros ha resultado enriquecedor y gratificante. Igualmente, supone una mayor motivación profesional a la vez que un mayor índice de satisfacción a la vista de los resultados obtenidos. Así, hemos tenido una escasa incidencia de complicaciones, a excepción de las 3 pacientes (11\%) que presentaron inestabilidad de novo, claramente independiente del régimen de ambulatorización o no del proceso, y que respondieron satisfactoriamente a la administración de anticolinérgicos orales.

Por todo ello, concluimos que la práctica totalidad de las pacientes afectas de IUE pueden ser tratadas mediante la colocación de un TVT bajo anestesia local-sedación y en régimen ambulatorio, considerando que la realización de este procedimiento bajo anestesia raquídea o general y con ingreso hospitalario constituyen una sobreindicación terapéutica que debe ser evitada.

\section{BIBUOGRAFIA y LECTURAS \\ RECOMENDADAS (*lectura de interés y **lectura fundamental)}

1. MORAL, L.; ALONSO, M.: "Impacto de la Cirugía Mayor Ambulatoria en España. Manual práctico de Cirugía Mayor Ambulatoria". 9 - 22; Ed Doyma SA; Madrid; 1999.

2. Ministerio de Sanidad y Consumo. Dirección General de Aseguramiento y Planificación Sanitaria. Cirugía Mayor Ambulatoria: Guía de Organización y Funcionamiento. Madrid, 1993.

3. DE LATHOWER, C.; POUILLIER, J.P.: "How much ambulatory surgery in the world in $1966-1977$ and trends". Amb. Surg.,. 8: 191, 2000.

4. JARRET, P.; DE LATHOWER, C.; OGG, T.W.: "The time has come to promote true day surgery". Amb. Surg., 8: 163, 2000.

**5. RODRÍGUEZ, J.M.; RODRÍGUEZ, R.; BLANCO, G.: "Cirugía Mayor Ambulatoria en Urología". Porrero JL. Cirugía Mayor Ambulatoria 2a Edición; 311-333; Ed Doyma SA; Madrid; 2002. 
**6. NAVALON, P.; ZARAGOZA, C.; CANOVAS, J.A. y cols.: "Cirugía Mayor Ambulatoria en Urología. Análisis de nuestra experiencia". Arch. Esp. Urol., 57: 513, 2004.

**7. LLOPIS, B.; NAVARRO, J.A.; MOLA, M.J. y cols.: "Cirugía mayor ambulatoria en urología: 5 años de experiencia". Actas Urol. Esp., 27: 117, 2003.

8. Generalitat Valenciana. Conselleria de Sanitat: "Guía de actuación en Cirugía Mayor Ambulatoria". Valencia, 2002.

9. DELANCEY, J.O.L.: "Structural support of the urethra as it relates to stress urinary incontinence". Am. J. Obstet. Gynecol., 170: 1713, 1994.

10. NICNEJAD, K.; PLZAK, L.S.; STASKIN, D.R. y cols.: "Cabestrillos uretrales autólogos y sintéticos para la incontinencia femenina". Urol. Cl. N. Amer., 3: 607, 2002.

11. LÓPEZ, E.; SÁNCHEZ, F.; NAVALON, P. y cols.: "Nuevos procedimientos quirúrgicos para el tratamiento de la incontinencia de esfuerzo. Su aplicación en cirugía mayor ambulatoria". Cir. May. Amb., 8: 178, 2003.

12. PETROS, P.; ULMSTEN, U.: "An integral theory of female urinary incontinence. Experimental and clinical considerations". Acta Obstet. Gynecol. Scand., 153: 7 , 1990.

*13. GALMÉS, I.; DÍAZ, E.: “¿Son iguales todos los sistemas empleados para corregir la incontinencia urinaria mediante mallas libres de tensión?". Actas Urol. Esp., 28: 487, 2004.

14. LO, T.S.; WANG, A.C.; HORNG, S.G. y cols.: "Ultrasonographic and urodynamic evaluation after tension-free vaginal tape (TVT)". Acta Obstet. Gynecol. Scand., 80: 65. 2001.

**15. GUTIERREZ, J.L.; MARTIN, B.; PORTILLO, J.A. y cols.: "Tratamiento de la incontinencia urinaria de esfuerzo mediante el implante suburetral de cintas libres de tensión (TVT). 4 años de experiencia”. Arch. Esp. Urol., 55: 885, 2003.

16. MUTONE, N.; MASTROPIETRO, M.; BRIZENDINE, E. y cols.: "Effect of tension free vaginal tape procedure on urodynamic continence indexes". Obstet. Gynecol., 98: 638, 2001.

17. SADIQ, A.; MANUNTA, A.; CHOGRANI, S. y cols.: "TVT colposuspension without preoperative urodynamic studies". Eur. Urol., 4: 15, 2005.

**18. GUTIERREZ, J.L.; MARTIN, B.; PORTILLO, J.A. y cols.: "Utilidad de la determinación de la presión abdominal de fuga en el diagnóstico de la incontinencia de orina femenina en la época del TVT ". Actas Urol. Esp., 28: 506, 2004.

19. NILSON, C.G.; KUUVA, N.; FALCONER, C. y cols.: "Resultados a largo plazo del procedimiento con cinta vaginal sin tensión para el tratamiento quirúrgico de la incontinencia urinaria de esfuerzo femenina". Gomelsky A, Dmochowski RR: Practical Cases in Urology. Self study program; 73-76; Ed Medical Trends SL; Barcelona; 2004.

*20. JIMÉNEZ, J.; HUALDE, A.; SANTIAGO, A. y cols.: "TVT: 3 años de experiencia". Actas Urol. Esp., 28: 13, 2004.

21. ULMSTEN, U.; HENRIKSSON, L.; JONHSON, P. y cols.: "An ambulatory surgical procedure under local anesthesia for treatment of female urinary incontenen- ce”. Int. Urogynecol. J. Pelvic. Floor Dysfunc., 7: 81, 1996.

22. RYU, K.H.; SHIN, J.S.; DU, J.K. y cols.: "Randomized trial of tension-free vaginal tape (TVT) vs tension free vaginal tape obturator (TVT-O) in the surgical treatment of stress urinary incontinence. Comparison of operation related morbidity". Eur. Urol., 4: 15, 2005.

23. THIEL, R.: "Retropubic vs transobturatoric approach (TVT vs TOT) for the treatment of stress incontinence". Eur. Urol., 4: 15, 2005.

**24. LIAPIS, A.; BAKAS, P.; CREATSAS, G.: "Burch colposuspension and tension-free vaginal tape in the management of stress urinary incontinence in women". Eur. Urol., 41: 469, 2002.

25. QUIEVY, A.; COUTURIER, F.; PRUDHON, C. y cols.: "Economic comparison of 2 surgical techniques for the treatment of stress urinary incontinence in women Burch's technique versus TVT technique". Prog. Urol., 11: 347, 2001.

26. LEBRET, T.; LUGAGNE, P.M.; HERVÉ, J.M. y cols.: "Evaluation of tension-free vaginal tape procedure". Eur. Urol., 40, 453: 2001.

27. JOMAA, M.: "Combined tension-free vaginal tape and prolapse repair under local anaesthesia in patients with symptoms of both urinary incontinence and prolapse". Gynecol. Obstet. Invest., 51, 184: 2001.

28. DEVAL, B.; JEFFRY, L.; AL NAJJAR, F. y cols.: "Determinants of patient dissatisfaction alter a tensionfree vaginal tape procedure for urinary incontinence". J. Urol., 167: 2093, 2001.

29. MUKHERJJE, K.; CONSTANTINE. G.: "Urinary stress incontinence in obese women: tension-free vaginal tape is the answer". BJU International, 88: 881 , 2001.

30. AZAM, U.; FRAZER, M.I.; KOZMAN, E.L. y cols.: "The tension-free vaginal tape procedure in women with previous failed stress incontinence surgery". J. Urol., 166: 554, 2001.

31. LO, T.S.; HORGN, S.G.; CHANG, C. y cols.: "Tension-free vaginal tape procedure after previous failure in incontinence surgery”. Urology, 60: 57, 2002.

32. DAVIS, J.E.: "Futuro de la Cirugía mayor ambulatoria”. Clin. Quir. Nort., 4: 921, 1987.

33. ELIZANDE, A.; REGOJO, O.; NAVARRO, J. y cols.: "Urología en un programa de Cirugía Mayor Ambulatoria". Actas Urol. Esp., 28, 5: 144, 2004.

34. CALDAMONE, A.; RABINOWITZ, R.: "Outpatient orchiopexy". J. Urol., 143: 951, 1990.

35. SADLER, G.; RICHARDS, H.; WATKINS, G. y cols.: "Day-case paediatric surgery: the only choice". Ann. R. Coll. Surg. Engl., 74: 130, 1982.

36. VARGAS, C.; RIUS, G.: "Cirugía urológica sin ingreso". Actas Urol. Esp., 17: 87, 1993.

*37. ESCRIBANO, G.; HERNANDEZ, C.; SUBIRA, D. y cols.: "Incontinencia urinaria de esfuerzo femenina. Corrección quirúrgica con técnicas de cabestrillo pubovaginal". Arch. Esp. Urol., 55: 1093, 2002.

*38. LEACH, G.E.: "Local anesthesia for urologic procedures". Urology, 48: 248, 1996.

39. SAMPIETRO, A.; VAQUERIZO, A.; FERNÁNDEZ, A. y cols.: "Cirugía mayor ambulatoria en Urología: nuestra experiencia”. Arch. Esp. Urol., 48: 343, 1995. 


\section{COMENTARIO EDITORIAL}

En los últimos años las disfunciones del suelo pélvico en la mujer han experimentado cambios relevantes en su interpretación y por tanto en el tratamiento de la incontinencia urinaria con o sin prolapso pélvico asociado. A pesar de la alta prevalencia de estos problemas, no eran frecuentes las comunicaciones de los trabajos de los distintos equipos de urólogos. Desde que surgió la teoría integral del suelo pélvico femenino, bien resumida por los autores de este trabajo, muchos profesionales hemos adquirido un renovado interés por estas patologías.

Seguro que en el futuro cercano se producirán, y así debe ser, la publicación de muchos trabajos sobre este tema.

Desde su descripción, y experiencias posteriores, han demostrado que la cirugía del cabestrillo suburetral para tratar la incontinencia urinaria de esfuerzo femenina, realizada en régimen ambulatorio, es una técnica factible, reproducible, eficaz y eficiente. A unque la serie de los autores es corta, el protocolo es riguroso y de ahí el buen resultado del mismo.

A veces las características del medio laboral de otros profesionales impiden que puedan desarrollar programas similares. De todas formas es la tendencia actual y su implantación en los distintos servicios será imparable.

De los apartados del trabajo comentaría que llama la atención el poco volumen de anestésico local usado, a pesar de usar la vía retropúbica. Q uizás por eso precisaron neuroleptoanalgesia con drogas potentes en la tercera parte de los pacientes. También la vía transobturador, en nuestra experiencia, es factible con similar protocolo anestésico.

En cuanto a dejar la sonda y el tapón durante 24 horas, será conveniente explorar su retirada en la misma unidad de recuperación. Seguramente, su retirada precoz, no van a impedir un problema de vaciado 0 un sangrado retropúbico importante en la mayoría de los casos, a la luz de lo publicado hasta ahora.

Me llama la atención las características y frecuencia de la urgencia miccional. Las 4 pacientes que tenían estos síntomas preoperatorios no mejoraron nada y si respondieron a anticolinérgicos. En nuestra experiencia y coincidiendo con la mayoría, mejoran mucho el $70-80 \%$ de las pacientes. En 3 pacientes la urgencia de novo está dentro de recogido por muchos, y también responden favorablemente a anticolinérgicos.

Creemos con los autores que en la mayoría de los casos no se necesita estudio urodinámico completo. Pero, una paciente que se opera de incontinencia urinaria de esfuerzo femenina pensamos que es necesario realizar una flujometría valorable.

Estaremos atentos a los trabajos de este tipo y animamos a los autores de este a seguir en el camino emprendido.

Dr. Gregorio Escribano Patiño

Servicio de Urología

Hospital General Universitario G regorio Marañón

Madrid

\section{CONTESTACION AL COMENTARIO EDITORIAL}

No cabe duda que la creación de nuevas unidades de CMA en España es un hecho imparable, siendo cada vez mayor el número de servicios de urología que se integran en este sistema organizativo de la asistencia quirúrgica, y aunque somos conscientes de que las características del medio laboral de otros profesionales no siempre permiten desarrollar este tipo de programas, la tendencia actual es a la instauración de los mismos, y en un futuro no lejano, su implantación constituirá un imperativo tanto en el sistema sanitario público como privado.

Respecto a la consideración acerca de los anestésicos locales, es obvio que un mayor volumen de fármaco administrado puede permitir una mayor superficie de tejido infiltrada, pero esto también supone una menor concentración del medicamento por su mayor dilución y por tanto un menor poder anestésico. Creemos que una concentración al 1\% de lidocaína proporciona un efecto anestésico suficiente siempre y cuando la aguja del TVT se deslice por el mismo recorrido por donde previamente pasó la aguja de infiltración depositando el fármaco, siendo también de gran importancia esperar el tiempo de latencia pertinente para que la solución anestésica difunda a los tejidos 
adyacentes ampliando la zona anestesiada. A simismo, nunca empleamos más de 30 cc de lidocaína $1 \%$, ya que ésta dosis, junto con la que se pudiera haber absorbido a través de la mucosa vaginal y uretrovesical del anestésico tópico aplicado en forma de gel, especialmente en pacientes de poco peso, puede fácilmente alcanzar la dosis tóxica (concentraciones plasmáticas $>6 \mu \mathrm{g} / \mathrm{ml}$ ). A sí, en caso necesario, antes de recurrir a aumentar la dosis de anestésico local, preferimos utilizar sedoanalgesia adicional con perfusión de propofol y remifentanilo, fármacos con efecto hipnótico y analgésico respectivamente (que no neuroleptoanalgésico) de rápido efecto y precoz eliminación, ampliamente utilizados por muchos grupos en cirugía ambulatoria.

N osotros también hemos empleado con éxito la vía del transobturador, a la que solo le vemos la ventaja de no soler precisar de cistoscopia. No obstante, encontramos dificultosa la infiltración anestésica de la cara interna del orificio obturador, por ser necesaria una mayor curvatura de la aguja de infiltración, lo que no siempre es posible realizar.

Por otro lado, nosotros también consideramos improcedente la utilización sistemática de sonda y tapón vaginal, de lo que habitualmente prescindimos en las pacientes intervenidas con ingreso hospitalario, pero en CMA deben extremarse todas las medidas para minimizar riesgos de complicaciones, por lo que creemos, al menos en nuestra experiencia, que su práctica representa una morbilidad mínima proporcionando cierta seguridad.

Dr. Pedro N avalón Verdejo 